\title{
Role of I-Scan in the Diagnosis of Patients with Portal Hypertensive Gastropathy
}

\author{
AYMAN YOUSY, M.D.*; HANY SHEHAB, M.D.*; KHALED RAGAB, M.Sc.**; \\ MAGED EL-GHANAM, M.D.**; MOHAMED GODA, M.D.**; HAYTHAM ABDALLA, M.D.**; \\ TAREK ABOUSHOUSHA, M.D.*** and NOHA HELAL, M.D.*** \\ The Departments of Endemic Medicine, Hepatology \& Gastroenterology*, Faculty of Medicine, Cairo University, \\ Hepatology \& Gastroenterology** and Clinical Pathology***, Theodor Bilharz Research Institute
}

\begin{abstract}
Background: Portal Hypertensive Gastropathy (PHG) occurs as a complication of cirrhotic or non-cirrhotic portal hypertension. PHG is clinically important because it might cause acute or chronic blood loss. The aim of the current study was to test the value of I-scan to predict gastric histologic diagnosis especially in cases with portal hypertensive gastropathy.

Subject and Methods: 113 subjects involved into this study and divided into two groups; Group (1) Consisted of twelve normal subjects while Group (2) Consisted of $101 \mathrm{HP}$ negative PHG. Upper endoscopy was performed for all cases, first by WLE then we shifted to I-scan technology, two biopsies were taken (one from greater curvature of the body of stomach and the other from antrum). Also full clinical assessment, biochemical profile, viral markers and ultrasound were performed for all the patients.
\end{abstract}

Results: PHG group showed a congested SECN and CV of the body and antrum with high specificity and sensitivity above $90 \%$.

Conclusion: I-scan has good sensitivity and specificity regarding diagnosis of PHG and poor specificity in the diagnosis of HP gastritis.

Key Words: Portal hypertensive gastropathy - I-scan.

\section{Introduction}

PORTAL Hypertensive Gastropathy (PHG) occurs as a complication of cirrhotic or non-cirrhotic portal hypertension. PHG is clinically important because it might cause acute, massive, or insidious blood loss. It is characterized by an endoscopic abnormality of the gastric mucosa that is classically described as a mosaic-like pattern that resembles the skin of a snake, with or without red spots [1]

Correspondence to: Dr. Ayman Yousy, The Department of Endemic Medicine, Hepatology \& Gastroenterology, Faculty of Medicine, Cairo University
The diagnosis of PHG is established when the characteristic endoscopic findings are observed in patients with portal hypertension [2]. Portal hypertensive gastropathy has a snake skin or mosaic background mucosa. The severity of PHG is associated with flat or bulging red or brown spots that might have friability or frank bleeding [3]. Narrow band imaging and I-scan are more sensitive than conventional white light endoscope for detection of mild PHG. The narrow band imaging system is an endoscopic imaging technique for the enhanced visualization of mucosal microscopic structure and capillaries of the superficial mucosal layer, by changing the spectral features of the illumination used in the video endoscope system [4].

\section{Subjects and Methods}

A total of adult 113 patients of both gender were included in this study who were presented to the Endoscopy Unit, Theodor Bilharz Research Institute (TBRI), Giza, Egypt and BVU Endoscopy Unit, Istanbul, Turkey. The study was carried out from January 2014 till November 2016. Ethical approval was obtained. The informed consents were obtained from all the patients recruited in the study. The age of the included patients ranged from 18-70 years old. All the included patients had an indication for upper endoscopy (i.e. dyspepsia) and any patients with active hematemesis or melena or previous gastric surgery were excluded.

The included patients were classified into two equal groups based on the histological finding; Group (1) formed of 12 patients with normal histological finding while Group (2) formed of 101 patients with PHG histology. 


\section{Procedure:}

A full history, clinical examination and childpugh grading for cirrhotic patients were performed for all the patients. Also a complete blood picture, ESR, hepatic profile (serum bilirubin, ALT, AST, alkaline phosphatase, serum albumin, prothrombin time and concentration). Renal biochemical profile, serum alpha fetoprotein and abdominal ultrasound (to detect liver texture, size, and the condition of the portal vein as well as the presence of Focal Hepatic Lesion (FHL) were performed.

For Upper endoscopy; all procedures were performed by a single endoscopist (conventional White Light Endoscopy (WLE) was used followed by I scan) and two gastric biopsies were taken, one from body and one from antrum. All procedures were recorded as high-definition video. I-scan examination was performed in two main sites: Gastric antrum and body. The I-scan endoscopy is HD image using PENTAX.

The criteria for diagnosing PHG by optical enhancement endoscopy (I-scan and NBI) appeared as extended and swollen gastric pits, with various degrees of dilated and convoluted capillaries surrounding the gastric pits. Red spots appeared as extended and swollen gastric pits, dilated and convoluted capillaries surrounding the gastric pits plus intra-mucosal hemorrhage around capillaries [5].

After finishing the examination; two biopsies one from the antrum and the other from body greater curvature were taken and preserved in formalin for histological examination. The observed mucosal and vascular pattern by I-scan were correlated with the histopathological results. Fifty of recorded videos were reviewed by an expert endoscopist and inter- observer agreement was calculated.

\section{Statistical methods:}

Data were coded and entered using the statistical package SPSS Version 21. Data was summarized using mean, standard deviation, median, minimum and maximum for quantitative variables and frequencies (number of cases) and relative frequencies (percentages) for categorical variables. Comparison of quantitative variables was done using the nonparametric Kruskal-Wallis when comparing more than 2 groups and using the non-parametric MannWhitney U-test when comparing 2 groups. Comparison between the different study groups using chi-square tests. $p$-value $<0.05$ was significant.

\section{Results}

Body SECN were present in $91.7 \%$ of normal group. Congested SECN present in $93.7 \%$ severe PHG \& $86.8 \%$ mild PHG, with a significant $p$ value according to chi square test.

Table (1): Body subepithelial capillary net work (SECN).

\begin{tabular}{|c|c|c|c|c|}
\hline & Normal & \multicolumn{2}{|l|}{ Mild PHG } & Severe PHG \\
\hline \multicolumn{5}{|l|}{ Body SECN: } \\
\hline \multicolumn{5}{|l|}{ Absent: } \\
\hline Count & 1 & \multicolumn{2}{|l|}{5} & \\
\hline$\%$ & $8.3 \%$ & \multicolumn{2}{|l|}{$13.2 \%$} & $6.3 \%$ \\
\hline \multicolumn{5}{|l|}{ Present: } \\
\hline Count & 11 & 0 & \multicolumn{2}{|r|}{0} \\
\hline$\%$ & $91.7 \%$ & \multicolumn{2}{|l|}{$0.0 \%$} & $0.0 \%$ \\
\hline \multicolumn{5}{|l|}{ Congested: } \\
\hline Count & 0 & 33 & \multirow{2}{*}{\multicolumn{2}{|c|}{$\begin{array}{l}59 \\
93.7 \%\end{array}$}} \\
\hline$\%$ & $0.0 \%$ & $86.8 \%$ & & \\
\hline \multirow[t]{3}{*}{ Total } & \multirow[t]{3}{*}{12} & 38 & \multicolumn{2}{|r|}{63} \\
\hline & & \multicolumn{3}{|c|}{ Chi-Square Tests } \\
\hline & & Value & df & $p$-value \\
\hline Pearson Chi-Square & & $326.090(a)$ & 8 & 0.000 \\
\hline Likelihood Ratio & & 332.770 & 8 & 0.000 \\
\hline $\mathrm{N}$ of Valid Cases & & 113 & & \\
\hline
\end{tabular}

Antral SECN were seen $91.7 \%$ normal group, congested SECN in $84 \%$ PHG group, with a significant $p$-value.

Table (2): Antral Sub Epithelial Capillary Net work (SECN).

\begin{tabular}{|c|c|c|c|c|}
\hline & Normal & Mild PHG & & Severe PHG \\
\hline \multicolumn{5}{|l|}{ Antrum SECN: } \\
\hline Count & 1 & 6 & \\
\hline$\%$ & $8.3 \%$ & $15.8 \%$ & \multicolumn{2}{|r|}{$7.9 \%$} \\
\hline \multicolumn{5}{|l|}{ Present: } \\
\hline Count & 11 & 0 & \multicolumn{2}{|r|}{0} \\
\hline$\%$ & $91.7 \%$ & $0.0 \%$ & \multicolumn{2}{|r|}{$0.0 \%$} \\
\hline \multicolumn{5}{|l|}{ Congested: } \\
\hline Count & 0 & 32 & \multicolumn{2}{|r|}{58} \\
\hline$\%$ & $0.0 \%$ & $84.2 \%$ & & $92.06 \%$ \\
\hline \multirow[t]{3}{*}{ Total } & 12 & 38 & \multicolumn{2}{|r|}{63} \\
\hline & & \multicolumn{3}{|c|}{$\begin{array}{l}\text { Chi-Square Tests between } \\
\text { pathological groups }\end{array}$} \\
\hline & & Value & df & $p$-value \\
\hline \multicolumn{2}{|l|}{ Pearson Chi-Square } & 381.48 & 16 & 0.000 \\
\hline \multicolumn{2}{|l|}{ Likelihood Ratio } & 355.142 & 16 & 0.000 \\
\hline \multicolumn{2}{|l|}{$\mathrm{N}$ of Valid Cases } & 113 & & \\
\hline
\end{tabular}


Body CV is present in $91.7 \%$ of normal cases and congested in $81.6 \%$ in mild PHG, $92.1 \%$ in severe PHG.

Table (3): Body collecting venules.

\begin{tabular}{|c|c|c|c|c|}
\hline & Normal & Mild PHG & & Severe PHG \\
\hline \multicolumn{5}{|l|}{ Body CV: } \\
\hline \multicolumn{5}{|l|}{ Absent: } \\
\hline Count & 1 & 7 & & 5 \\
\hline$\%$ & $8.3 \%$ & $18.4 \%$ & & $7.9 \%$ \\
\hline \multicolumn{5}{|l|}{ Present } \\
\hline \multicolumn{5}{|l|}{ (not congested): } \\
\hline Count & 11 & 0 & & 0 \\
\hline$\%$ & $91.7 \%$ & $0.0 \%$ & & $0.0 \%$ \\
\hline \multicolumn{5}{|l|}{ Congested: } \\
\hline Count & 0 & 31 & & 58 \\
\hline$\%$ & $0.0 \%$ & $81.6 \%$ & & $92.1 \%$ \\
\hline \multirow[t]{2}{*}{ Total } & 12 & 38 & & 63 \\
\hline & \multicolumn{2}{|c|}{ Value } & df & $p$-value \\
\hline Pearson Chi-Square & \multicolumn{2}{|c|}{ 335.291(a) } & 8 & 0.000 \\
\hline Likelihood ratio & \multicolumn{2}{|c|}{323.064} & 8 & 0.000 \\
\hline $\mathrm{N}$ of valid cases & \multicolumn{3}{|l|}{113} & \\
\hline
\end{tabular}

From the data represented in Table (4) 5 patients had normal gastric mucosa by WLE and on switching to I-scan technique the capillaries surrounding gastric pits on the antral mucosa were dilated and convoluted which classified them as mild PH. No statistical significant difference between WLE and I-scan $(p<.48)$. Inter-observer agreement was calculated, 95\% agreement in mucosal pit pattern and $88 \%$ in vascular pattern.

Table (4): Difference between WLE and I scan in the diagnosis of PHG.

\begin{tabular}{ll}
\hline Variables & Patients diagnosed \\
\hline PHG by WLE: & \\
No PHG & 5 \\
Mild PHG & 33 \\
Sever PHG & 63 \\
\hline Total cases diagnosed PHG by WLE & $96 / 101$ \\
PHG by I scan: & \\
No PHG & 0 \\
Mild PHG & 38 \\
Sever PHG & 63 \\
\hline Total cases diagnosed PHG by I scan & $101 / 101$ \\
\hline
\end{tabular}

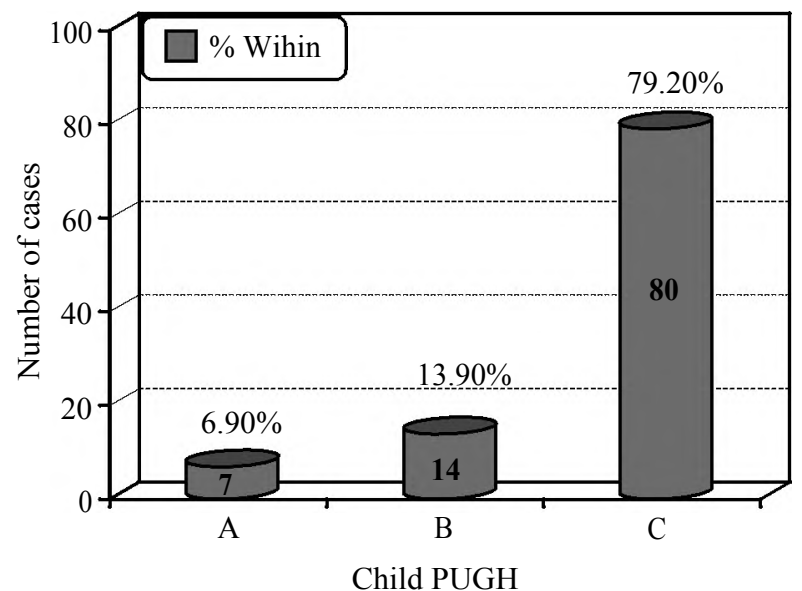

Fig. (1): $80 \%$ of patients in the PHG group were child C, $6.9 \%$ child A and $13.9 \%$ child $B$.

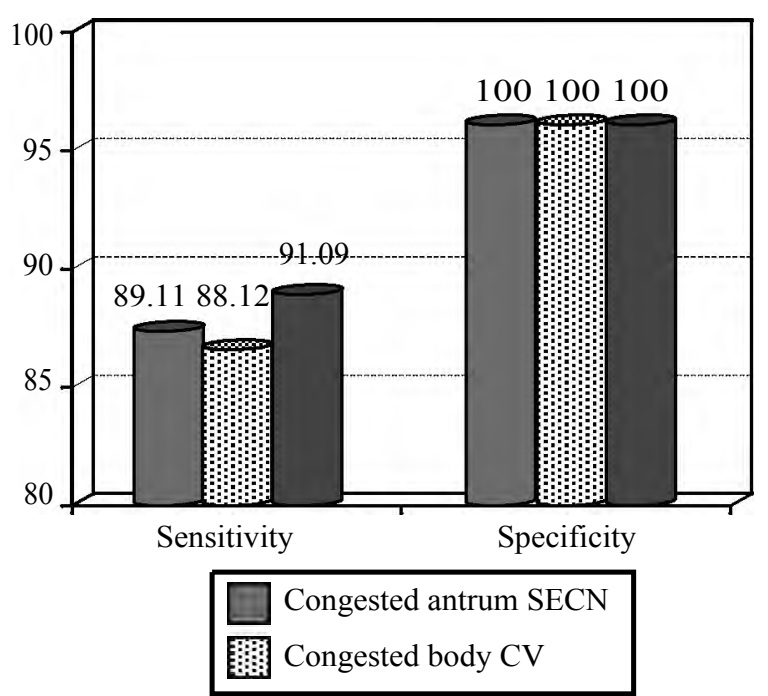

Fig. (2): The sensitivity of congested antrum SECN, body $\mathrm{CV}$ and body SECN in diagnosis of PHG were $89.1 \%$, $88.12 \%$ and $91.1 \%$ respectively the specificity of congested antrum SECN and body CV and SECN in the diagnosis of PHG was $100 \%$.

\section{Discussion}

The current study revealed that Child's classification was significantly higher in (PHG group) this goes in agreement with [6] who had performed a study on patients with liver cirrhosis and reported that variables significantly associated with $\mathrm{PHG}$ were history of gastrointestinal bleed, ascites, high bilirubin, deranged prothrombin time, higher variceal grade, high Child-Pugh score, and high hepatic venous pressure gradient, this finding disagree with Pan et al., [7] who reported that the most important element causing PHG is the increased portal pressure as a prerequisite. In addition, other factors may contribute to the development of PHG. PHG often occurs in patients with the presence of esophageal varices, the development 
of PHG is less influenced by the severity of liver disease. The current study is descriptive and tried to show vascular abnormalities and mucosal changes in the stomach and their correlation with histology. We showed that all cases of PHG had edematous enlarged rounded pattern in the body and edematous tubular pattern in the antrum with different degrees of congested CV and SECN. In cases of severe PHG there were also red spots. Hayashi and Saeki [5] made the same observation but they were working with NBI. In our study we found that the sensitivity and specificity of I-scan to diagnose PHG was $88.1 \%, 100 \%$ for congested SECN and $91.1 \%, 100 \%$ for congested CV respectively. The findings of congested SECN and CV were similarly described in two descriptive studies El-Shazly et al. [4] and Hayashi and Saeki, [5] using NBI.

In this study White Light Endoscopy (WLE) had the same sensitivity and specificity as I scan in detection of severe PHG but in case of mild PHG, I scan detected more cases of mild PHG yet with no significant statistical difference. This result is matching with El-Shazly et al. [4], in his study they showed that WLE and NBI detected the same number of cases in severe PHG while NBI detected a slightly higher number of mild PHG (four patients) which were missed by WLE. These finding also highlight the low utility of I-scan (in comparison to WLE) in the diagnosis or staging of PHG.
As complications of PHG are related to its severity, the detection of mild form of PHG by Iscan will not add benefit as regards to the prognosis.

\section{References}

1- THULUVATH P.J. and YOO H.Y.: Portal hypertensive gastropathy. Am. J. Gastroenterol., 97 (12): 2973-8, 2002.

2- RIPOLL C. and GARCIA-TSAO G.: Management of gastropathy and gastric vascular ectasia in portal hypertension. Clinics in liver disease, 14 (2): 281-95, 2010.

3- PATWARDHAN V. and CARDENAS A.: Review article.The management of portal hypertensive gastropathy and gastric antral vascular ectasia in cirrhosis. Alimentary Pharmacology \& Therapeutics, 2014.

4- EL-SHAZLY Y.M., SAID E., RAFIK M.M., et al.: Narrow band imaging: A new tool for diagnosis of portal hypertensive gastropathy. Nature \& Science, 12 (6), 2014.

5- HAYASHI S. and SAEKI S.: Endoscopic microvascular architecture of the portal hypertensive gastric mucosa on narrow band imaging. Digestive Endoscopy, 19: 116-23, 2007.

6- KUMAR A., MISHRA S.R. and SHARMA P.: Clinical, laboratory, and hemodynamic parameters in portal hypertensive gastropathy: A study of 254 cirrhotics. Journal of Clinical Gastroenterology, 44 (4): 294-300, 2010.

7- PAN W.D., XUN R.Y. and CHEN Y.N.: Correlations of portal hypertensive gastropathy of hepatitis B cirrhosis with other factors. Hepatobiliary Pancreat Dis. Int. Nov., 1 (4): 527-30, 2002.

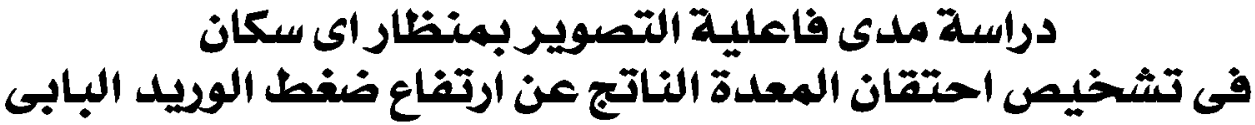

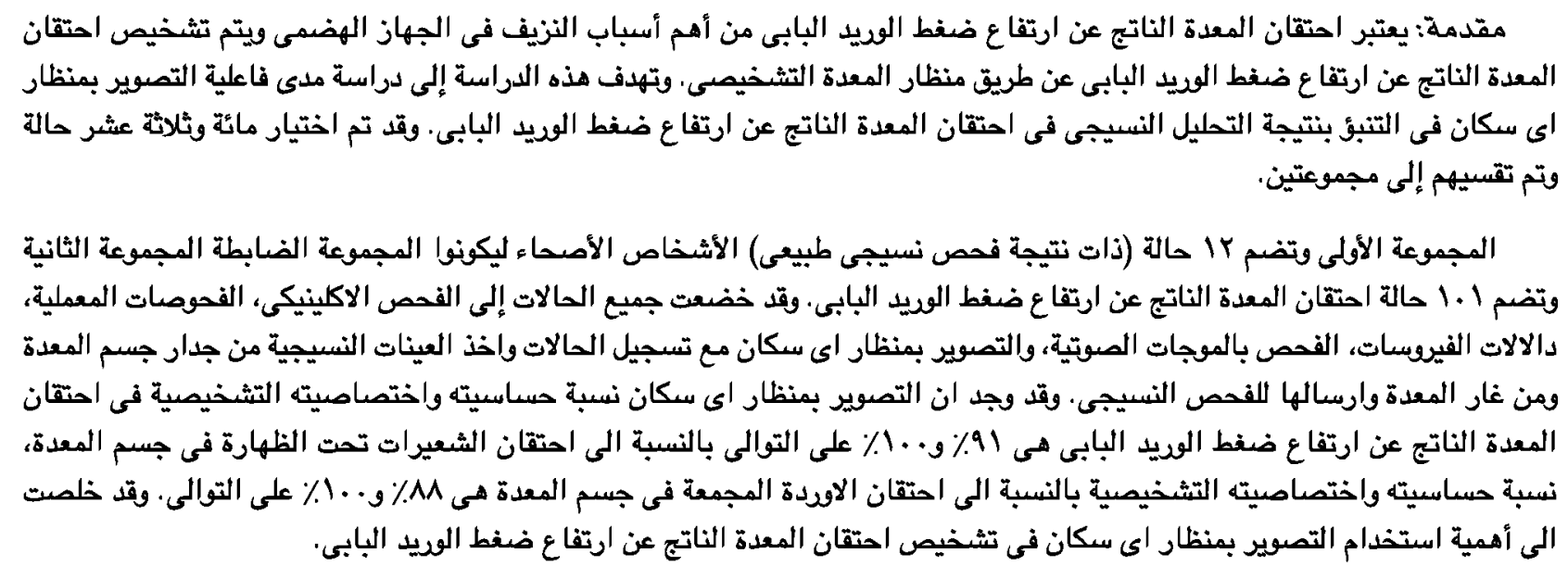

